\title{
Asymptomatic protozoa community infections in humans from southwestern China
}

\author{
Li-Guang Tian \\ China CDC NIPD \\ Cristina Ballesteros \\ UAB \\ Jia-Xu Chen \\ China CDC NIPD \\ Yan-Hong Chu \\ China CDC NIPD \\ Ying-Fang $\mathrm{Yu}$ \\ China CDC NIPD \\ Xiu-Ping Wu \\ China CDC NIPD \\ Xue-Jiao Teng \\ China CDC NIPD \\ Yi-Ting Li \\ China CDC NIPD \\ Emmanuel Serrano
}

UAB

Shun-Xian Zhang ( $\square$ zhangshunxian110@163.com )

Chinese CDC https://orcid.org/0000-0002-8929-4433

Zhen-Hui Lu

Shanghai Longhua hosptial

Lan-Lan Geng

Guangzhou Woman and children's medical center

Research article

Keywords: Co-Infection, Gastrointestinal disease, Human, Enteric protozoa, China

Posted Date: April 30th, 2019

DOI: https://doi.org/10.21203/rs.2.9410/v1 
License: (c) (i) This work is licensed under a Creative Commons Attribution 4.0 International License. Read Full License 


\section{Abstract}

Background: Giardia.lamblia (G.lamblia), Entamoeba histolytica (E.histolytica), Blastocyistis hominis (B. hominis ) and Cryptosporidium spp. infections have been frequently reported as etiological agents for gastroenteritis but also as common gut inhabitants in healthy individuals. Co-infections with protozoa pathogens were previously described but little is known about whether these assemblages are purely random or structured in communities. Methods From 1st July 2016 to 31st March 2017, fresh stool samples were collected from randomly selected individuals in sentinel hospitals in Tengchong City, Yunnan Province, China. molecular biology method was used to detect G.lamblia, E.histolytica, Cryptosporidium spp and B. hominis. Sequencing was applied to confirm the these protozoon genotypes. The data analysis method was involved chi-square test, multivariable logistic regression, null models and Partial least square regression (PLSR) methods with R 3.2 software. Results: The prevalence of the four enteric protozoa in all subjects were (in order of frequency of detection) : B.hominis 9.5\% [95\%Confidence Interval (Cl) 7.1-12.4\%], G.lamblia 2.2\% (95\% Cl 1.1-3.8\%); E.hystolitica 2.0\% (95\% Cl 0.9-3.6\%) whereas Cryptosporidium spp. was not detected at all. The prevalence of, at least, one enteric protozoa was $12.4 \%$ (95\% $\mathrm{Cl}$ 9.7-15.6), and the prevalence of 2 different enteric protozoa species was $1.2 \%(95 \% \mathrm{Cl} 0.4-2.6)$. The most common co-infection found was E.histolytica and B.hominis, (1.0\%; 95\% Cl 0.3-2.2). Regarding genetic profiles, 10 out of $11 \mathrm{G}$.lamblia strains were classified in assemblage A, sub-assemblage $\mathrm{I}$, and 1 sample was classified in assemblage B, sub-assemblage IV. B.hominis was detected in 48 samples, 25 were classified as genotype III, 13 as genotype I, 8 samples as genotype VII and the 2 remaining isolates as genotype IV. Our null modelling confirmed the random protozoa co-occurrence and our PLSR analysis the lack of association between these enteric pathogens and clinical symptomatology. Conclusions: The occurrence of these enteric protozoa was purely random. Not specific interactions were detected between the four protozoa studied and neither their presence, jointly or separately, nor the patient's age were predictors for developing clinical symptoms associated with these pathogens. Further research including a broader range of pathogen species is needed to address remaining knowledge gaps in co-infections and diarrhoeal disease.

\section{Background}

Parasitic disease has been frequently reported as a significant etiological agent of gastrointestinal disorders and its contribution to the burden of diarrhoeal diseases is really significant with several enteric protozoan species described as causative agents in humans[1-5].

Cryptosporidium spp., Entamoeba histolytica (E.histolytica), Giardia.lamblia (G.lamblia) and Blastocyistis hominis ( $B$. hominis ) can be considered as the four main enteric protozoa commonly associated with gastrointestinal disorders $[5,6]$. However, the impact that these parasites have on public health has not been fully characterized yet and the information available depends on the protozoa species in question. Thus, it is estimated that around $20 \%$ of diarrhoeal episodes in children in low and middle-income countries (LMIC) and 9\% in high-income settings (HIC) are caused by Cryptosporidum spp.[1]. Amebiasis, the acute disease caused by E.hystolitica, affects around 50 million people and causes 100,000 deaths 
each year[7]. Both protozoa, E.histolytica and Cryptosporidum spp., were included as causative of diarrhoeal deaths in the Global Burden Disease Study of 2017[4]. On the other hand, G.duodenalis infection is estimated to result in 280 million cases annually worldwide [8] and was included in the "Neglected Disease Initiative", together with Cryptosporidum spp. in 2006[9]. Regarding B.hominis, although it has been the commonest enteric protozoa isolated from patients with diarrhoeal diseases in $\mathrm{HIC}[1]$, its pathogenicity remains controversial[10,11] partly because it is the most common enteric protozoa detected in asymptomatic individuals also[12]. However it is have been argued that this protozoa could be used as an indicator of potential exposure to other pathogenic enteric protozoa[13].

Interestingly, the presence of any of these four protozoan species in the stools of asymptomatic individuals is not exclusive of $B$.hominis, and is relatively common according to some studies $[1,14,15]$ but there is a lack of reliable data due to the absence of monitoring programs, under-reporting $[16,17]$ and the fact that carrier and subclinical stages are often not diagnosed $[1,17]$.

Even though there have been notable advances, a lack of understanding about what are the factors that determine the development of gastrointestinal symptoms as a result of enteric protozoan infection still persists[18]. Protozoan genotype has been suggested as one of the most decisive predictors, although not the only one. It is expected that the growing development of new diagnostic tools, particularly molecular techniques, contributes to clarify the distinction between pathogenic and non-pathogenic lineages as well as pathophysiological interactions and other epidemiological features of interest [19].

Many previous studies have explored and reported the presence of more than one pathogen in diarrhoeal disease cases $[6,19,20]$ as well as in healthy individuals [21, 22], but only a few of them have been addressed to explore the impact of concomitant enteric infections and to characterize the enteric communities and the existence of specific interactions $[15,23]$. These interactions, at least between 2 specific enteric pathogens, are well documented in the veterinary field, such as the association of enterotoxigenic Escherichia coli and rotavirus that lead to severe diarrhoea in piglets as an example [24] but it has not been addressed in immunocompetent humans until recently $[15,25]$.

In order to understand the existence and impact of intestinal protozoan co-infections in humans, we performed a hospital-based cross-sectional study. The aims of the study were: 1) to investigate the prevalence of the four protozoa species most commonly associated with gastrointestinal disorders and determine their molecular profile, 2) to assess whether enteric protozoa in hospital-based patients cooccur by chance or are structured in a community, and 3) to explore the potential impact of co-infections with the detected species on the clinical symptomatology among immunocompetent patients attending the People's Hospital of Tengchong City and the Chinese Medicine Hospital in Tengchong City.

\section{Methods}

\section{Study design and study area}


A cross-sectional hospital-based study was conducted from July 2016 to March 2017 in Tengchong $\left(25^{\circ} 01^{\prime} 15^{\prime \prime} \mathrm{N}, 98^{\circ} 29^{\prime} 50^{\prime \prime} \mathrm{E}, 1596 \mathrm{~m}\right.$ above sea level) a county level city located in Yunnan Province,

Soutwest China. Tengchong has a tropical monsoon climate, the annual average temperature is $15^{\circ} \mathrm{C}$ and the average annual rainfall is $1535 \mathrm{~mm}$ with a year-round mean relative humidity of $77 \%$. The total resident population is 659,000 (Census 2014) of which $60.5 \%$ live in rural areas. Two hospitals from this city (People's Hospital of Tengchong City and Chinese Medicine Hospital of Tengchong City) agreed to participate in the study.

The target sample size was 423 for an expected prevalence of $50 \%, 95 \%$ confidence interval and $5 \%$ precision. Finally, 507 patients were recruited.

\section{Study participants}

Patients from inpatient department were voluntary recruited after a clear explanation of the study project objectives provided by the researcher. Informed consent was obtained from the participants or their parents, and participant's confidentiality was assured. Subjects with inadequate fecal samples, incomplete information, and those patients infected with Hepatitis B virus (HBV) and/or Human Immunodeficiency virus (HIV) were excluded.

\section{Specimen and data collection}

Stool samples were collected from each subject with a sterile sampling cup during the study period in these two hospitals, with the criterion that each stool must be greater than $3 \mathrm{~g}$ or $3 \mathrm{~mL}$, and stored at $-70^{\circ} \mathrm{C}$. All the samples were delivered without interrupting the cold chain to the laboratory of National Institute of Parasitic Diseases, Chinese Centre for Disease Control and Prevention (Shanghai, China) and stored in a $-70^{\circ} \mathrm{C}$ refrigerator.

A structured questionnaire was used to collect the following information from each patient after the stool samples were collected: demographic data (e.g. age, gender, level of education, occupation, nationality and residence) and clinical manifestations associated with enteric protozoa infection (abdominal distension, inappetence, itchy skin, perianal pruritus, constipation, nausea, abdominal pain, , number of stools per day and type of stools).

\section{Detection of intestinal protozoan species}

Genomic DNA was extracted from each stool sample (0.2 gram or 0.2 milliliter) with QIAamp DNA stool mini kit (Qiagen, Hilden, Germany) according to the manufacturers' protocol. The genomic DNA was stored at $-70^{\circ} \mathrm{C}$ until polymerase chain reaction (PCR) amplification (Table 1). It was amplified by conventional PCR for B. hominis [26], and nested PCR for Cryptosporidium spp., G.lamblia and E.histolytica [27-29].

\section{Genetic characterization of G.lamblia and B.hominis isolates}


All suspicious positive products were kept at $-70^{\circ} \mathrm{C}$, and bidirectional sequencing by Sangon Biotech Company (Shanghai, China) was conducted to confirm the accuracy of G.lamblia and B.hominis infections. The nucleotide sequences obtained in this study were aligned with the G.lamblia amd B.hominis reference nucleotide sequences from GenBank and analyzed with BLAST (http://www.ncbi.nlm.nih.gov/BLAST/), and MEGA version 6.0 (http://www.megasoftware.net/) to determine G.lamblia assemblages and B.hominis subtypes based on Neighbour-Joining method. The reference nucleotide sequences of G.duodenalis from GenBank for TPI included assemblages AI (accession numbers L02120 and EF68803), All (accession numbers U57897 and EF688019), All (accession number DQ650648), E (accession number JN162352), C (accession number JF958102), D (accession numbers DQ246216 and DQ220289), BIII (accession numbers AY228628 and AY069561), BIV (accession number AY368371). And the B.hominis reference nucleotide sequences from GenBank ST1(U51151), ST2(AB070997, AB070987), ST3(AB070988, AB070986), ST4(AY244621, U51152), ST5(AB070999, AB107966), ST6(AB070990, AB070994), ST7(AF408427, AB070991), ST8(AY266472), ST9(AY266470), ST10(KC148207, KX352008), ST11(GU256903, GU256904), ST12(GU256905), ST13(GU256935) and ST14(KC148206).

\section{Statistical modelling}

Data were analyzed using the R 3.2 software. The chi-square or fisher exact test, Odd ratios (OR) and 95\% confidence intervals $(95 \% \mathrm{Cls})$ were used to compare and described the qualitative variables.

Only participants with complete data records were included in the final analysis. A new variable was created according to the WHO's definition of diarrhoea and the information gathered about type of stool and number of stools per day. A case of acute diarrhoea was defined as a person with more than three episodes of liquid stools per day, lasting less than 2 weeks [30]. Prevalences at $95 \%$ confidence intervals (Cls) for single infections and co-infections in the study population were calculated using epiR library version $0.5-10[31]$.

\section{Co-occurrence of enteric pathogens}

A null model analysis was used to explore whether enteric protozoa co-infection were positive, negative, or randomly associated. Data were organized as a presence-absence $4 \times 507$ (row $\times$ columns) matrix, in which each row represented a protozoa species and each column represented a study participant, "1" indicated that a species was present at a particular host and " 0 " indicated that a species was absent.

The C-score was the co-occurrence index used for co-occurrence patterns characterization and the algorithm chosen was the fixed row-equiprobable column [32] The calculated C-score was compared with the expected C-score calculated for 5000 randomly assembled null matrices by Monte Carlo simulations. Furthermore, to compare the degree of co-occurrence across data, a standardised effect size (SES) was calculated, an index that measures the number of standard deviations that the observed index (C-score) is above or below the mean index of the simulated communities. The package EcoSimR 0.1.0 was used to carry out the analysis[33]. 


\section{Assessing the impact of co-infection with enteric pathogens on diarrhoea severity}

The partial least square (PLS) regression method was used to assess the impact of co-infection with enteric protozoa on the development of clinical symptomatology. This technique was selected as it offers multiple advantages over other regression methods: it is the least restrictive of the multivariate techniques for exploring complex ecological patterns[34], including the impact of co-infections on the host's health[23] and its distribution is free and well suited to deal with multicollinearity[35]. In our analysis, we defined explanatory and response components or blocks. The explanatory block (PLS X's component) was defined by a presence-absence matrix representing the enteric protozoa community ( $B$. hominis, E.histolytica, Criptosporidium sp., and G.lamblia). In addition, due to the previously mentioned age variability in the clinical presentation of diarrhoeal diseases, age in years was also included as a covariate in the explanatory block. Our response block (PLS's Y component) included the main symptoms described associated with the infections of those protozoa (abdominal distension, inappetence, itchy skin, perianal pruritus, constipation, nausea, abdominal pain and acute diarrhoea).

The significance of PLS models was assessed using the Stone-Geisser's Q2 test, a cross-validation redundancy measure created to evaluate the predictive significance of exogenous variables. Values greater than 0.0975 indicate that predictors are statistically significant, whereas values below this threshold reveal no significance. Finally, the percentage of observed MNLS variability explained by the enteric pathogen block was also estimated. The plspm library version 0.4 .9 was used to perform the analysis[36].

\section{Results}

\section{Characteristics of the study population}

A total of 507 subjects participated in the study. The male-to-female sex ratios was 1.1 (260/247). Han nationality was predominant with 481 participants $(94.9 \%)$ and the median age of study participants was 52 years (Interquartile range $(\mathrm{IQR})=38-63)$.

Most of the subjects lived in rural areas 78.1\% (396/507). Around two thirds of them had completed primary education $(65.6 \%, 332 / 507)$ and $63.1 \%$ of the participants were farmers $(320 / 507)$.

The most common clinical symptom was inappetence $(17.9 \%, 91 / 507)$, followed by abdominal pain $(16.6 \%, 84 / 507)$ and nausea $(16.6 \%, 84 / 507)$, other symptoms were: acute diarrhoea $(5.5 \%, 28 / 507)$, abdominal distension $(12.4 \%, 63 / 507)$, itchy skin $12.2 \%, 62 / 507)$, constipation $(10.8 \%, 55 / 507)$ and perianal pruritus $(5.5 \%, 28 / 507)$. $269(53.1 \%)$ patients did not have any gastrointestinal symptom.

\section{Single enteric pathogen infections and co-infections}

G.lamblia was detected in 11 patients, prevalence of $2.2 \%$ (95\% Cl 1.1-3.8\%); E. hystolitica was found in 10 patients, prevalence of $2.0 \%(95 \% \mathrm{Cl} 0.9-3.6 \%)$ and B.hominis was detected in 48 patients, prevalence of $9.5 \%$ (95\% Cl 7.1-12.4\%). Cryptosporidium sp. was not detected at all. 
The detection rate of E.hystolitica in diarrhea cases was higher than that of in non-diarreah subjects $(11.9 \%, 7 / 59.0 .7 \%, 3 / 448$. OR=19.9, 95\% Cl 5.0-79.5\%), and the prevalence of G.lamblia in diarreha patients was higher than that of in controls $(6.8 \%, 4 / 59.1 .6 \%, 7 / 448$. OR=4.6, 95\% Cl 1.3-16.2\%), but no significant difference of the $B$.hominis infection was observed among diarrhea cases and subjects $(13.6 \%, 8 / 59.8 .9 \%, 40 / 448 . \mathrm{OR}=1.6,95 \% \mathrm{Cl} 0.7-3.6 \%)$.

At least one enteric protozoa was detected in 63 patients $(12.4 \% ; 95 \% \mathrm{Cl} 9.7-15.6)$, and 2 different enteric protozoa species were detected in 6 patients $(1.2 \% ; 95 \% \mathrm{Cl} 0.4-2.6)$. The most common co-infection found was E.histolytica and B.hominis, detected in 5 patients $(1.0 \% ; 95 \% \mathrm{Cl} 0.3-2.2)$. Along the same lines, G.duodenalis and B.hominis co-infection was detected in 1 patient.

\section{Genetic characterization of isolates}

A total of 11 G.lamblia isolates were successfully characterized (Figure 1), revealing 10 strains were assemblage $A(90.9 \%, 10 / 11)$, and one stain was assemblage $B(9.1 \%, 1 / 11)$. Further, all 10 assemblage A strains were Al sub-assemblage, and the only one assemblage B strain was BIV sub-assemblage. The detection rate of assemblage $A$ in diarrhea cases was higher than that of in non-diarrhea subjects $(4 / 59$, 6/448, $P=0.02, \mathrm{OR}=5.4,95 \% \mathrm{Cl}: 1.5-19.6$ ).

B. hominis was detected in 48 samples, $25(52.1 \%, 25 / 48)$ samples were classified as genotype III, 13 $(27.1 \%, 13 / 48)$ as genotype I, $8(16.7 \%, 8 / 48)$ samples were genotype $\mathrm{VII}$ and a couple of samples(4.17\%, 2/48) were genotype IV (Figure 2). The prevalence of various subtypes of $B$. hominis was statistically different $\left(c^{2}=25.7045, P<0.0001\right)$ among individuals: the ST3 subtype $(4.93 \%, 25 / 507,95 \%$ Cl. $2.38-7.29)$ had the highest prevalence, followed by ST1 (2.56\%, 13/507, 95\% Cl: 1.43-4.45), ST7 (1.58\%, 8/507, 95\% Cl: $0.74-3.21)$ and ST4 $(0.39 \%, 2 / 507,95 \%$ Cl: $0.07-1.57)$.

\section{Impact of co-infection on diarrhoeal symptomatology}

According to our PLS analyses, there was no evidence that the presence of any of the three protozoa species detected or their co-occurrence were statistically associated with enteric protozoa symptomatology (Stone-Geisser's Q2 test value $<0.0975$ ). Regarding the other predictor, age, it was not statistically associated with symptomatology in the study population either.

Furthermore, the presence of any of the three protozoa, jointly or separately, covaried negatively with symptomatology. The analysis revealed that most of the X's component variance was due to co-infection with enteric protozoa (43.1\%), followed by B.hominis (27.6\%) and E.histolytica presence (24.0\%) (Table 2).

\section{Co-occurrence of enteric protozoa}

The null model analysis showed that the observed C-score (265) was lesser than expected by chance (267.45), indicating the existence of a random, non-competitively structured protozoa community. There 
was not evidence of a statistically significant protozoa combination (SES -0.33, p-value 0.414 ) in the study population.

\section{Discussion}

Our study confirms the single prevalence of enteric protozoa reported in other studies in similar settings $[1,17,37]$ with adequate access to water, sanitation and hygiene practices (WASH). In the present report, B.hominis was the predominant intestinal protozoa species detected followed by E.histolytica and G.lamblia whereas Cryptosporidium spp. was not detected at all, these data are consistent with previous results[5].

Regarding the genetic profile of the isolates, $11 \mathrm{G}$.lamblia strains were classified as assemblage A, within those isolates sub-assemblage $A I$ was dominant $(10,90.9 \%)$ and no mixed co-infection of $A$ and $B$ genotype was found. This result was consistent with those found in other countries [38] and in Anhui Province, China. In the latter, A subtypes accounted for $100 \%$ of G.lamblia isolated from asymptomatic children[39].

The presence and proportion of assemblages seems to presents spatiotemporal variations $[40,41]$ although socio-economic factors have been also suggested as potential drivers for G.lamblia assemblage distribution [22]. Interestingly, assemblage's variance seems to be involved in clinical presentation and only assemblages $A$ and $B$ have been described as causative agents of human infection, with assemblage $B$ resulting more frequently in symptomatic infection in endemic settings [21, 42]. Regarding assemblage $A, A l$ has been one of the commonest sub-assemblage reported in the literature although All is considered the most pathogenic sub-assemblage in humans [17, 43, 44]. All of the above could provide a potential explanation about why no evidence of association was found between the presence of G.lamblia and clinical symptomatology in the present study.

Regarding B.hominis, four genotypes were identified among 48 isolates. 25 isolates belonging to genotype III, 13 to genotype I, 8 were classified as genotype VII and a couple of samples were identified as genotype IV. These data are in agreement with other studies, who reported subtype III as the commonest $[45,46]$. As it has been previously highlighted, its clinical significance remains unclear[10].

As we have shown, it seems that, at enteric protozoan level, there is not any specific community assemblage in the study population considering the four protozoan species considered.

Our work showed that the occurrence of those enteric protozoan species was purely random and asymptomatic in the study population. Furthermore, not specific interactions were detected between them and there was no evidence that their presence, jointly or separately, was associated with the development of clinical symptomatology classically associated with those species. Although all of them have been reported as causative agents of gastrointestinal disorders, especially in children in LMIC[20], asymptomatic carrier has been also commonly reported elsewhere and, even in some cases such as B.hominis, its pathogenicity is subject to a big controversy $[10,11]$ since this species is one of the most 
common enteric protozoa detected in humans $[12,46]$, so it is not surprising that their presence was not associated with clinical symptomatology in immunocompetent individuals.

Given the results obtained, different factors might provide an explanation that help to understand the pathogenesis of enteric protozoan infections and what are the conditions that lead to disease. Some of them are related to the characteristics of the pathogen, such as different pathogenicity due to virulence variability of strains or the need of, at least, a second infection with another pathogen to cause clinical symptomatology. Moreover, host factors can play a key role too such as individual susceptibility or the presence of healthy functional barriers that protect the human intestine: the mucus layer, the epithelial intestinal layer and the intestinal microbioma $[5,15,18,25]$. However, further research needs to be performed to establish the ecology of enteric communities in healthy and unhealthy individuals.

We are aware that our research may have some limitations. Firstly, it was a hospital-based crosssectional study so the study population may be not representative of the general population in Tengchong City and the generalizability to other populations may be rather limited. Although the number of protozoa isolates was consistent with previous studies, it was small; this might have influenced the reliability of the estimates in the PLS analyses.

Therefore, further research with larger sample size, which considers a broader enteropathogen community is needed to explore community assemblages present as well as their relationships with pathogenicity and clinical manifestations in rural and urban regions in China.

Undoubtedly, with the increasing awareness of the microbiome role in the development of host immunity [5] and their potential relationship with many communicable and non-communicable diseases such as Irritable Bowel Syndrome[47], there is a growing need to understand the complex relationships between different pathogens and as well as between the intestinal microbiota and pathogens.

\section{Conclusions}

The present study was the first to analyse community assemblage of the four protozoan species commonly associated with human gastrointestinal disorders in immunocompetent individuals. Our results showed the absence of any structured community between them, their occurrence was purely random. Moreover, there was no evidence of an association between their presence and the development of clinical symptomatology.

Further research including a broad range of enteric pathogens is needed to disentangle the complex relationships and interactions of the intestinal ecosystem. This could eventually lead to a better understanding about what are the drivers behind gastrointestinal disorders.

\section{Abbreviations}


B. Hominis: Blastocyistis hominis. Cl: Confidence Interva. E.histolytica: Entamoeba histolytica. HBV: Hepatitis B virus. G.lamblia: Giardia.lamblia. HIC: high-income settings. HIV: Human Immunodeficiency virus. LMIC: middle-income countries. IQR: Interquartile range. OR: Odd ratios. PCR: polymerase chain reaction. PLSR: Partial least square regression. SES: standardised effect size. WASH: water, sanitation

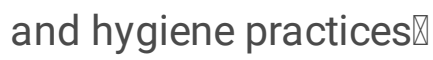

\section{Declarations}

\section{Acknowledgements}

We sincerely thank the efforts of the hospital in collecting stool specimens, and investigating and reporting on the subjects

\section{Funding}

The field epidemiological investigation and Blastocyistis hominis detection was supported by the National Natural Science Foundation of China (No.81473022), Giardia duodenalis, Entamoeba histolytica and Cryptosporidium spp. detection was supported by China Postdoctoral Science Foundation Grant (2018M64303043) and the postdoctoral research funding of SX-Z from Guangzhou Women and Children's Medical Center (5001-3001075). The data analysis was conducted by C. Ballesteros and E. Serrano. E. Serrano was funded by the Spanish Ministerio de Economia y Competitividad (MINECO) through a Ramon y Cajal agreement (RYC-2016-21120).

\section{Availability of data and materials}

Data of the study can be available upon request from the author (LG-T).

\section{Authors' contributions}

SX-Z, CB and ES performed the statistical analysis and drafted the manuscript, LG-T, LL-G ZH-L and JX-C conceived and designed the study, YH-C, YF-Y, XP-W, XJ-T and YT-L conducted the dates collected and pathogens detected. All authors read and approved the final version of the paper.

\section{Authors' information}

SX-Z, ZH-L and LL-G are the corresponding authors of this paper of this article. LG-T and CB contributed equally to this paper.

\section{Competing interests}

The authors declare that they have no competing interests.

\section{Consent for publication}

Not applicable. 


\section{Ethics approval and consent to participate}

The study was approved by the ethical review committee of the National Institute of Parasitic Diseases, Chinese Center for Disease Control and Prevention (No. 2014-004). Informed written consent was obtained from the subject or their parents/guardians before collecting the stool samples and information.

\section{Author details}

1 National Institute of Parasitic Diseases, Chinese Center for Disease Control and Prevention, Shanghai 200025, People's Republic of China; 2 Key Laboratory for Parasitology and Vector Biology, National Health Commission of the People's Republic of China, WHO Collaborating Center for Tropical Diseases, National Center for International Research on Tropical Diseases, Shanghai 20025, People's Republic of China. 3 Wildlife Ecology \& Health group (WE\&H), and Servei d'Ecopatologia de Fauna Salvatge (SEFaS), Departament de Medicina i Cirurgia Animals, Universitat Autònoma de Barcelona (UAB), Bellaterra, Spain. 4. Dipartimento di Scienze Veterinarie, Universitá di Torino, Grugliasco, Torino, Italy

5 Guangzhou Institute of Pediatrics, Guangzhou Women and Children's Medical Center, Guangzhou Medical University, Guangzhou 510623, People's Republic of China. 6 Department of Gastroenterology, Guangzhou Women and Children's Medical Center, Guangzhou Medical University, Guangzhou 510623, People's Republic of China. 7 Department of Respiratory, Longhua Hospital Shanghai University of Traditional Chinese Medicine, Shanghai 20025, People's Republic of China.

\section{References}

[1]. Fletcher, S.M., et al., Enteric protozoa in the developed world: a public health perspective. Clin Microbiol Rev, 2012. 25(3): p. 420-49.

[2]. Shrestha, A., et al., Intestinal parasite infections and associated risk factors among schoolchildren in Dolakha and Ramechhap districts, Nepal: a cross-sectional study. Parasit Vectors, 2018. 11(1): p. 532.

[3]. Hotez, P.J., et al., The global burden of disease study 2010: interpretation and implications for the neglected tropical diseases. PLoS Negl Trop Dis, 2014. 8(7): p. e2865.

[4]. Roth, G.A., et al., Global, regional, and national age-sex-specific mortality for 282 causes of death in 195 countries and territories, 1980-2017: a systematic analysis for the Global Burden of Disease Study 2017. The Lancet, 2018. 392(10159): p. 1736-1788.

[5]. Partida-Rodriguez, O., et al., Human Intestinal Microbiota: Interaction Between Parasites and the Host Immune Response. Arch Med Res, 2017. 48(8): p. 690-700.

[6]. Maas, L., et al., Detection of intestinal protozoa in paediatric patients with gastrointestinal symptoms by multiplex real-time PCR. Clin Microbiol Infect, 2014. 20(6): p. 545-50. 
[7]. Leung, P., et al., Epidemiological features of intestinal infection with Entamoeba histolytica in Taiwan, 2002-2010. Travel Medicine and Infectious Disease, 2014. 12(6): p. 673-679.

[8]. Esch, K.J. and C.A. Petersen, Transmission and epidemiology of zoonotic protozoal diseases of companion animals. Clin Microbiol Rev, 2013. 26(1): p. 58-85.

[9]. Savioli, L., H. Smith and A. Thompson, Giardia and Cryptosporidium join the 'Neglected Diseases Initiative'. Trends Parasitol, 2006. 22(5): p. 203-8.

[10]. Audebert, C., et al., Colonization with the enteric protozoa Blastocystis is associated with increased diversity of human gut bacterial microbiota. Sci Rep, 2016. 6: p. 25255.

[11]. Coyle, C.M., et al., Blastocystis: To Treat or Not to Treat. Clinical Infectious Diseases, 2012. 54(1): p. 105-110.

[12]. Fouad, S.A., et al., The pathogenic role of different Blastocystis hominis genotypes isolated from patients with irritable bowel syndrome. Arab J Gastroenterol, 2011. 12(4): p. 194-200.

[13]. Schaechter, M., Intestinal protozoan. In: Encyclopedia of microbiology. Oxford, United Kingdom: Academic Press. 2009: p. 696-705.

[14]. Olesen, B., et al., Etiology of diarrhea in young children in Denmark: a case-control study. J Clin Microbiol, 2005. 43(8): p. 3636-41.

[15]. Zhang, S.X., et al., Impact of co-infections with enteric pathogens on children suffering from acute diarrhea in southwest China. Infect Dis Poverty, 2016. 5(1): p. 64.

[16]. Newell, D.G., et al., Food-borne diseases - the challenges of 20 years ago still persist while new ones continue to emerge. Int J Food Microbiol, 2010. 139 Suppl 1: p. S3-15.

[17]. Horton, B., et al., Giardia duodenalis in the UK: current knowledge of risk factors and public health implications. Parasitology, 2019. 146(4): p. 413-424.

[18]. Levine, M.M. and R.M. Robins-Browne, Factors that explain excretion of enteric pathogens by persons without diarrhea. Clin Infect Dis, 2012. 55 Suppl 4: p. S303-11.

[19]. Boughattas, S., et al., Molecular Analysis of the Enteric Protozoa Associated with Acute Diarrhea in Hospitalized Children. Front Cell Infect Microbiol, 2017. 7: p. 343.

[20]. Kotloff, K.L., et al., Burden and aetiology of diarrhoeal disease in infants and young children in developing countries (the Global Enteric Multicenter Study, GEMS): a prospective, case-control study. Lancet, 2013. 382(9888): p. 209-22.

[21]. Jerez, P.L., et al., Molecular analysis of Giardia duodenalis isolates from symptomatic and asymptomatic children from La Habana, Cuba. Parasite Epidemiol Control, 2017. 2(3): p. 105-113. 
[22]. Ramirez, N.E., L.A. Ward and S. Sreevatsan, A review of the biology and epidemiology of cryptosporidiosis in humans and animals. Microbes Infect, 2004. 6(8): p. 773-85.

[23]. Risco, D., et al., Severity of bovine tuberculosis is associated with co-infection with common pathogens in wild boar. PLoS One, 2014. 9(10): p. e110123.

[24]. Tzipori, S., et al., Escherichia coli and rotavirus infections in four-week-old gnotobiotic piglets fed milk or dry food. Aust Vet J, 1980. 56(6): p. 279-84.

[25]. Serrano, E. and J. Millan, What is the price of neglecting parasite groups when assessing the cost of co-infection? Epidemiol Infect, 2014. 142(7): p. 1533-40.

[26]. Menounos, P.G., et al., Direct detection of Blastocystis sp. in human faecal samples and subtype assignment using single strand conformational polymorphism and sequencing. Mol Cell Probes, 2008. 22(1): p. 24-9.

[27]. Liu, H., et al., Prevalence and genetic characterization of Cryptosporidium, Enterocytozoon, Giardia and Cyclospora in diarrheal outpatients in China. BMC Infect Dis, 2014. 14: p. 25.

[28]. Sulaiman, I.M., et al., Triosephosphate isomerase gene characterization and potential zoonotic transmission of Giardia duodenalis. Emerg Infect Dis, 2003. 9(11): p. 1444-52.

[29]. Evangelopoulos, A., et al., A nested, multiplex, PCR assay for the simultaneous detection and differentiation of Entamoeba histolytica and Entamoeba dispar in faeces. Ann Trop Med Parasitol, 2000. 94(3): p. 233-40.

[30]. WHO, WHO Diarrhea. http://www.who.int/topics/diarrhoea/en/. 2018.

[31]. Aragon TJ, E., Epidemiology Tools. https://cran.r-project.org/package=epitools. 2017.

[32]. Gotelli, N., Null model analysis of species co-occurrence patterns. Ecology, 2000. 9(81): p. 26062621.

[33]. Nick Gotelli, E.H.A.E., Null model analysis for ecological data. https://github.com/gotellilab/EcoSimR. 2015.

[34]. Gordo, L.M.C.I., Partial least squares regression as an alternative to current regression methods used in ecology. OIKOS, 2009.

[35]. Goodier, J., Encyclopedia of Measurement and Statistics. 2007.

[36]. Gaston Sanchez, L.T.G.R., Tools for Partial Least Squares Path Modeling (PLS-PM). 2017.

[37]. Li, J., et al., Giardia duodenalis Infections in Humans and Other Animals in China. Frontiers in microbiology, 2017. 8: p. 2004 
[38]. Skhal, D., et al., Prevalence of Giardia duodenalis assemblages and sub-assemblages in symptomatic patients from Damascus city and its suburbs. Infect Genet Evol, 2017. 47: p. 155-160.

[39]. Yu, Y.F., et al., [Infection of Giardia lamblia in HIV-Infected Individuals and in Kindergarden Children in Rural Area of Anhui and Genotype Analysis]. Zhongguo Ji Sheng Chong Xue Yu Ji Sheng Chong Bing Za Zhi, 2016. 34(6): p. 537-41.

[40]. Bartelt, L.A. and R.B. Sartor, Advances in understanding Giardia: determinants and mechanisms of chronic sequelae. F1000Prime Rep, 2015. 7: p. 62.

[41]. Heyworth, M.F., Giardia duodenalis genetic assemblages and hosts. Parasite, 2016. 23: p. 13.

[42]. Hussein, E.M., et al., Nested PCR targeting intergenic spacer (IGS) in genotyping of Giardia duodenalis isolated from symptomatic and asymptomatic infected Egyptian school children. Parasitol Res, 2017. 116(2): p. 763-771.

[43]. Xiao, L. and R. Fayer, Molecular characterisation of species and genotypes of Cryptosporidium and Giardia and assessment of zoonotic transmission. Int J Parasitol, 2008. 38(11): p. 1239-55.

[44]. Štrkolcová, G., et al., Giardia duodenalis and Giardia enterica in children: first evidence of assemblages A and B in Eastern Slovakia. Parasitology Research, 2016. 115(5): p. 1939-1944.

[45]. Fouad, S.A., et al., The pathogenic role of different Blastocystis hominis genotypes isolated from patients with irritable bowel syndrome. Arab J Gastroenterol, 2011. 12(4): p. 194-200.

[46]. Yoshikawa, H., et al., Polymerase chain reaction-based genotype classification among human Blastocystis hominis populations isolated from different countries. Parasitol Res, 2004. 92(1): p. 22-9.

[47]. Cifre, S., et al., Blastocystis subtypes and their association with Irritable Bowel Syndrome. Med Hypotheses, 2018. 116: p. 4-9.

\section{Tables}

Table 1: Diagnostic techniques used to detect enteric pathogens in in Tengchong city, southwest China 


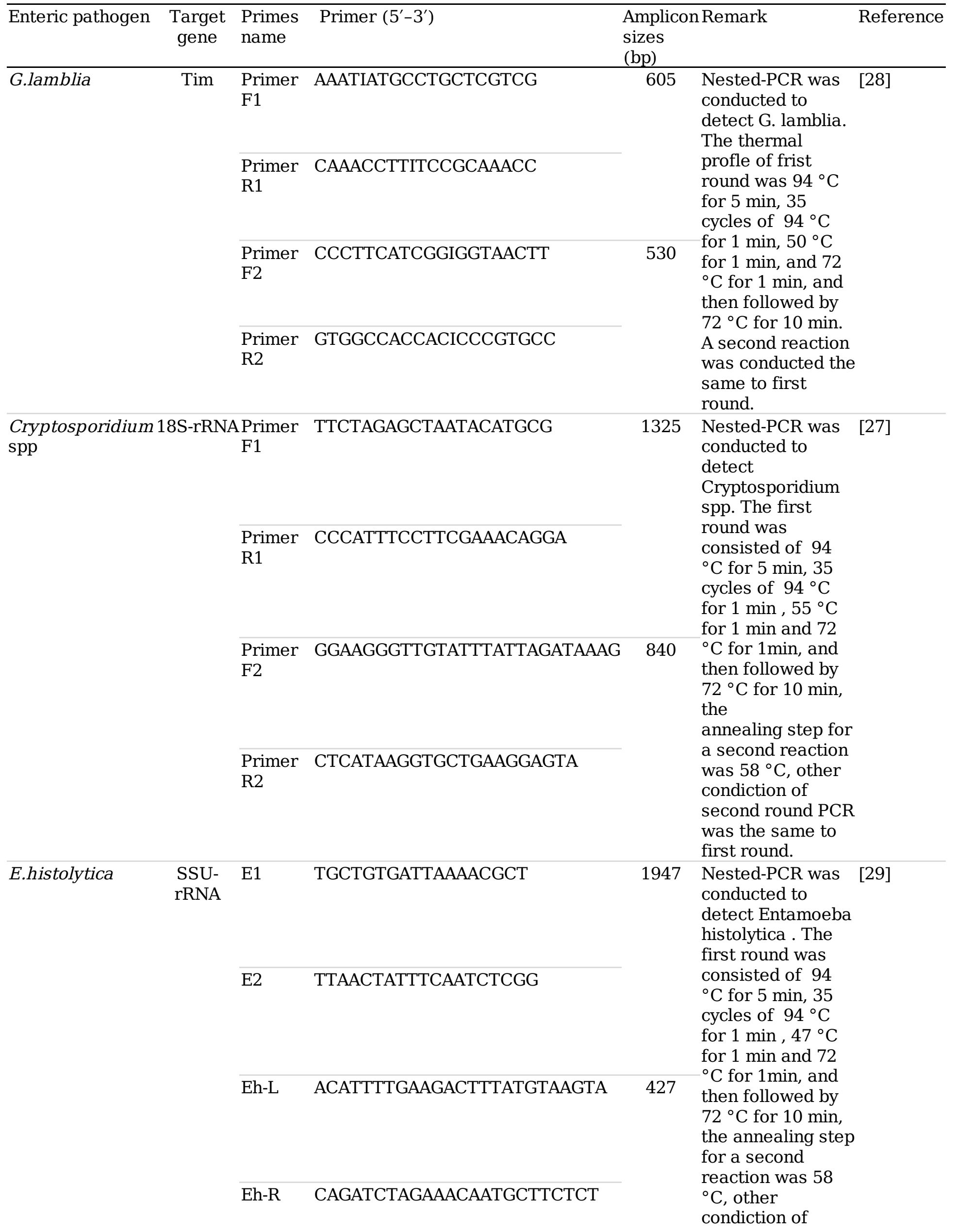


second round PCR was the same to first round.

\begin{tabular}{|c|c|c|c|c|}
\hline B. hominis & $\begin{array}{l}\text { SSU } \\
\text { rRNA }\end{array}$ & Reverse ACTAGGAATTCCTCGTTCATG & 1100 & $\begin{array}{l}\text { PCR was applied } \\
\text { to detect } \\
\text { Blastocystis spp, } \\
\text { The thermal } \\
\text { profle was } 94^{\circ} \mathrm{C} \\
\text { for } 5 \text { min, } \\
\text { followed by } 35 \\
\text { cycles of } 94^{\circ} \mathrm{C} \text { for } \\
1 \text { min, } 54^{\circ} \mathrm{C} \text { for } 1 \\
\text { min, and then } 72^{\circ} \\
\text { for } 1 \text { min. This is } \\
\text { followed by a final } \\
\text { annealing cycle of } \\
72^{\circ} \mathrm{C} \text { for } 10 \text { min. }\end{array}$ \\
\hline
\end{tabular}

Table 2: Predictor weights of the PLS model explaining their association with clinical symptomatology in hospital-based patients in Tengchong City

\begin{tabular}{lcccc}
\hline \multicolumn{5}{l}{ Predictor variables Loads Weights Percent Cross-correlation } \\
\hline Protozoa richness & -0.67 & -0.66 & 43.1 & -0.11 \\
\hline G. lamblia & -0.2 & -0.17 & 2.9 & -0.03 \\
\hline B. hominis & -0.56 & -0.53 & 27.6 & -0.08 \\
\hline E. histolytica & -0.44 & -0.5 & 24 & -0.08 \\
\hline Age & 0.1 & 0.15 & 2.2 & 0.02 \\
\hline
\end{tabular}

Figures 


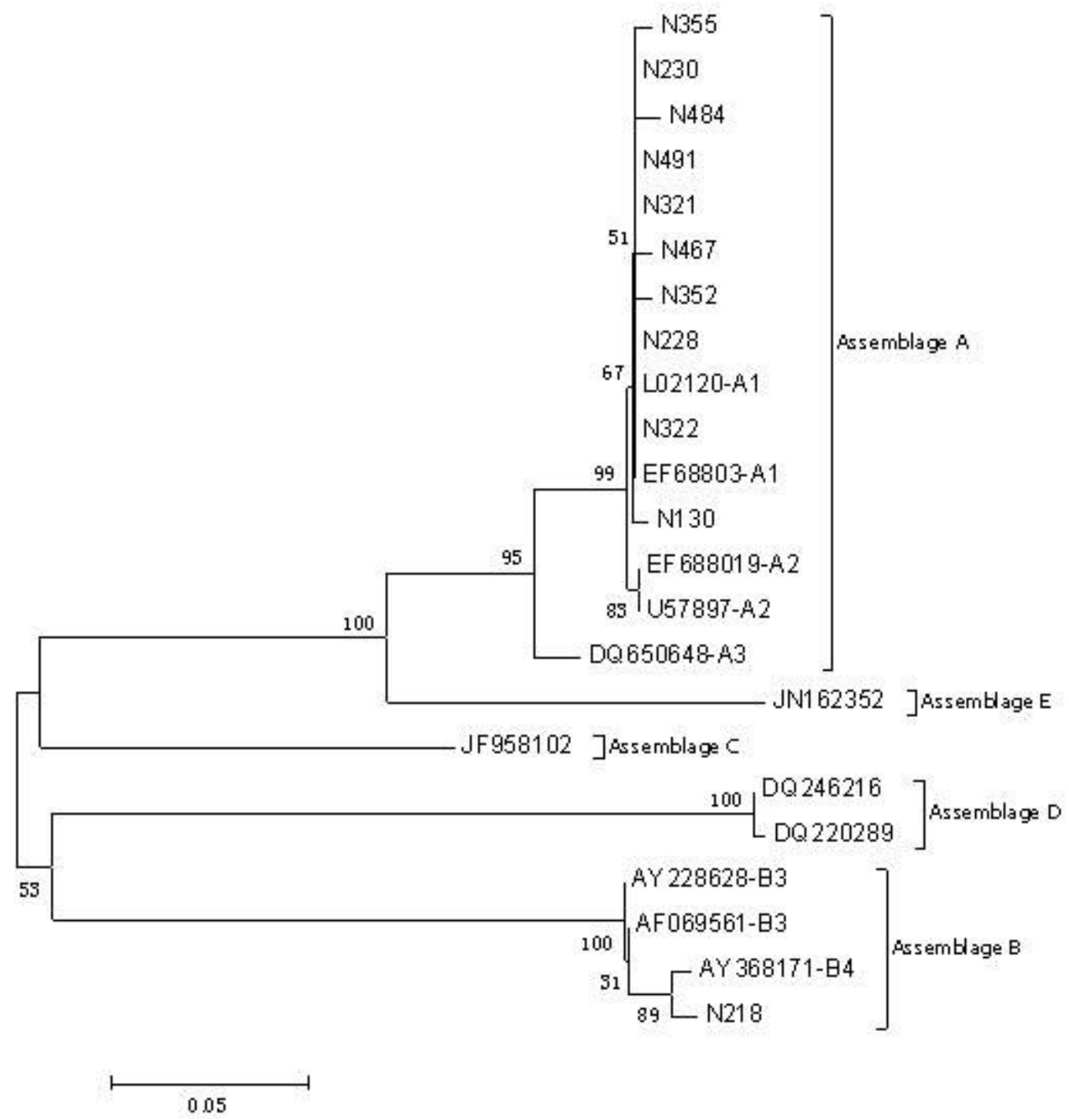

Figure 1

Evolutionary relationships among G. lamblia sub-assemblages at the TPI locus inferred by a NeighborJoining analysis. The percentage of replicate trees in which the associated taxa clustered together in the bootstrap test (1,000 iterations) is indicated next to the branches. N355, N230, N484, N491, N321, N467, N352, N228, N322 and N130 were isolated from human in this study and were all Al, and the N218 was BIV. 


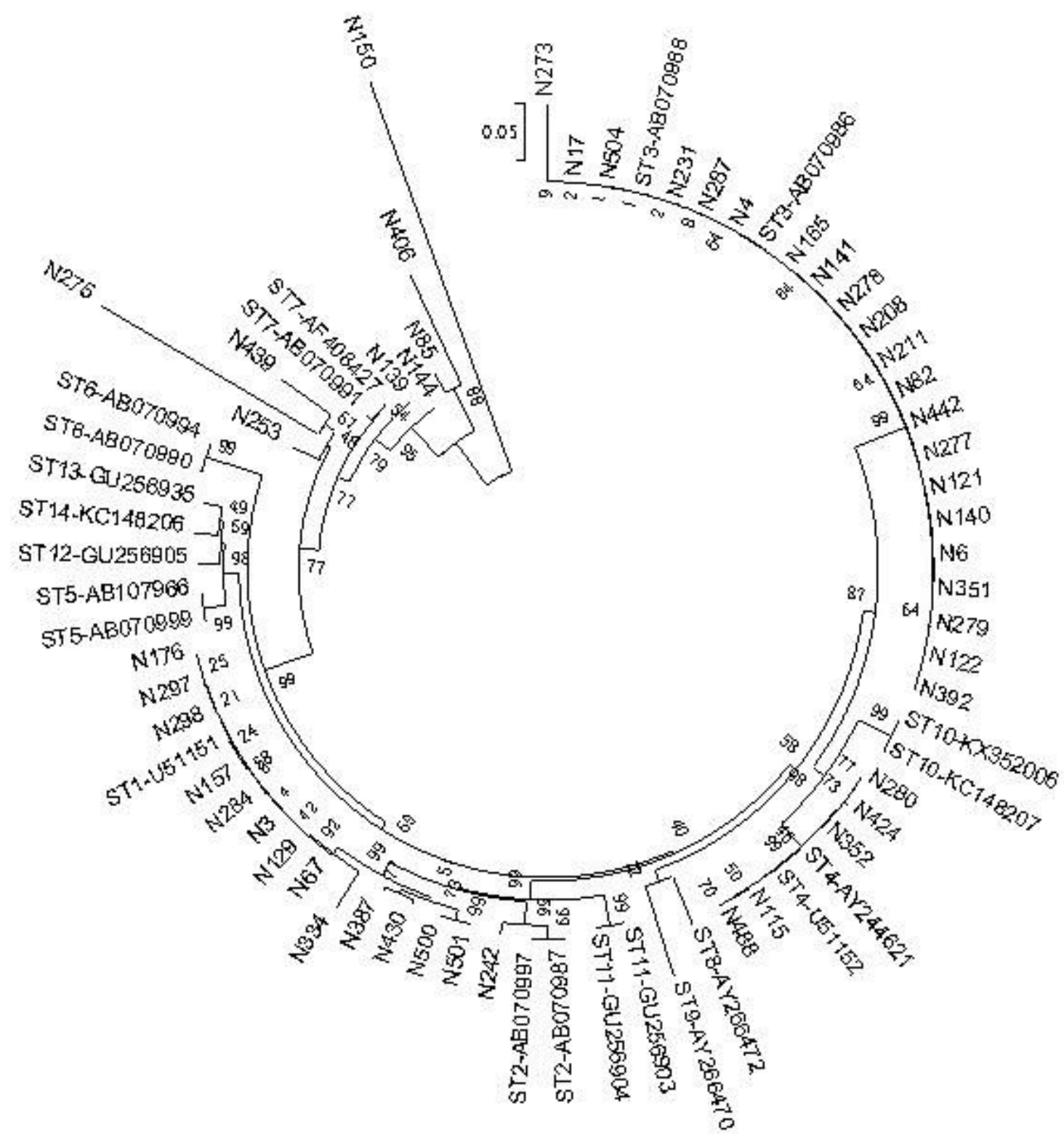

Figure 2

Evolutionary relationships among B. hominis genotypes by a neighbor-joining analysis. The percentage of replicate trees in which the associated clustered together in the bootstrap test (1,000 iterations) is indicated next to the branches. 